\title{
At the Vigintennial of the Butcher-Oemler Effect
}

\author{
Kevin A. Pimbblet \\ Department of Physics, University of Queensland, Brisbane, Queensland 4072, Australia \\ pimbblet@physics.uq.edu.au \\ Received 2003 June 16, accepted 2003 July 30
}

\begin{abstract}
In their study of the evolution of galaxies within clusters, Butcher and Oemler discovered evidence for a strong evolution in star-formation rate with redshift. Later studies confirmed this conclusion and uncovered several aspects of the effect: photometric, spectroscopic, and morphological. This article reviews a broad sample of these works and discusses selection effects, biases, and driving mechanisms that might be responsible for the changes in star-formation rate.
\end{abstract}

Keywords: galaxies: evolution — galaxies: clusters: general — cosmology: observations

\section{Introduction}

About twenty years ago, Butcher and Oemler published a series of influential papers investigating the evolution of galaxies within clusters of galaxies (Butcher \& Oemler 1978a, 1978b, 1984, 1985; Butcher, Wells, \& Oemler 1983). From this series, it is the 1984 paper that describes the Butcher-Oemler (BO hereafter) effect. To understand the $\mathrm{BO}$ effect, it is necessary to examine the observational foundations upon which it is built.

Clusters of galaxies allow the study of a large number of galaxies $\left(\sim 10^{3}\right)$ at a common distance and visible out to a large redshift, which makes them ideal for studying galaxy evolution. They feature predominantly passively evolving early-type (elliptical and lenticular) galaxies in their core regions (e.g. Pimbblet 2001). It is these galaxies that Visvanathan \& Sandage (1977) first noted exhibit systematically redder integrated colours than the late-type (spiral and irregular) galaxies in clusters. Further, the early-type galaxies also demonstrate a tight correlation between their colours and magnitudes: a colour-magnitude relationship (CMR hereafter: e.g. Bower, Lucey, and Ellis 1992). By calculating $R_{30}$, the projected clustocentric radius that contains 30 per cent of the total galaxy population, Butcher \& Oemler (1984) found the excess fraction of galaxies bluer than the CMR. Specifically, this blue fraction, $f_{B}$, is defined to be the fraction of galaxies within $R_{30}$ brighter than $M_{V}=-20$ whose rest frame $(B-V)$ colour is at least 0.2 magnitudes bluer than the cluster's CMR.

Importantly, they also uncovered evidence for an evolution in the blue fraction with redshift, increasing from $f_{B} \sim 0.03$ at low redshifts $(z \sim 0.05)$ to $f_{B} \sim 0.25$ at larger redshifts $(z \sim 0.5)$. It is this evolution that is commonly referred to as the $\mathrm{BO}$ effect. The implication of these results is that the star-formation rate within galaxies has decreased dramatically with epoch irrespective of local environment. This article presents a historical review of progress into the understanding of the origins of the BO effect. In Section 2, the confirmation of the BO effect is presented alongside some immediate complicating factors. Section 3 discusses attempts to tie together several strands of observational evidence (photometric, spectroscopic, and morphological), whilst in Section 4, possible mechanisms for the effect are discussed. How selection effects can alter the calculated values of $f_{B}$ are discussed in more detail in Section 5. Finally, some concluding remarks are made in Section 6.

\section{Confirmations and Complications}

The original work has been followed up by many other authors (e.g. Couch \& Newell 1984; Rakos \& Schombert 1995) who show that the cores of $z>0.2$ clusters do contain blue galaxies, complementing the work of other authors (e.g. Terlevich, Caldwell, \& Bower 2001) who find uniformly red, early-type galaxies populating local nearby clusters. The natural conclusion to this situation supports the original one: there has been strong, rapid evolution of clusters of galaxies with redshift.

More recently, Smail et al. (1998) examined a sample of 10 rich clusters of galaxies in the redshift range $0.22<z<0.28$ and found that the blue fraction (median $f_{B} \sim 0.05$ ) is lower than the values found by Butcher \& Oemler (1984; see Figure 1). The individual measurements, however, show a large scatter (see also La Barbera et al. 2003). There is also found to be no significant correlation between $f_{B}$ and global cluster parameters such as X-ray morphology or cluster concentration. Other studies (e.g. Margoniner et al. 2001; Pimbblet et al. 2002; Figure 1) display a similarly large scatter in $f_{B}$.

To test the cluster membership of these blue $\mathrm{BO}$ galaxies, a number of groups (e.g. Dressler \& Gunn 1983; Couch \& Sharples 1987) undertook spectroscopic surveys of distant clusters and came up with surprising results. Although some blue galaxies are found to be interloping foreground or background galaxies, the bona fide blue cluster members are found to exhibit spectra typical of spiral galaxies. They display strong emission lines indicative of ongoing star formation. Further, some red galaxies show strong Balmer absorption lines with a lack of emission lines, indicative of a recent decline in star formation (Figure 2). 


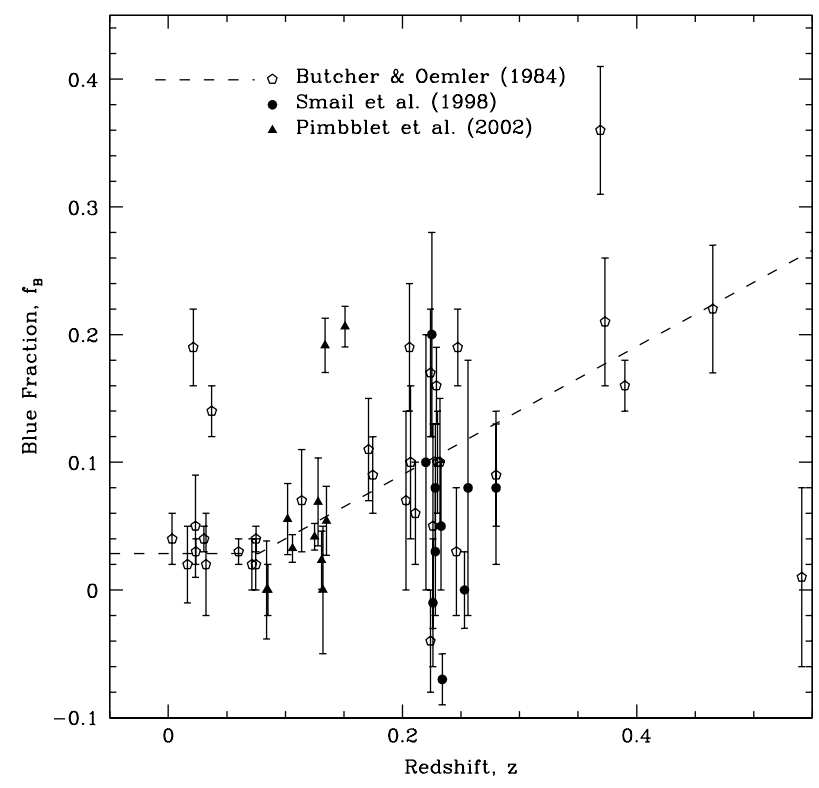

Figure 1 The Butcher-Oemler effect, showing an evolution in the blue fraction with redshift (dashed line). Also plotted are the points from Smail et al. (1998) (filled circles) and Pimbblet et al. (2002) (filled triangles) for comparison.

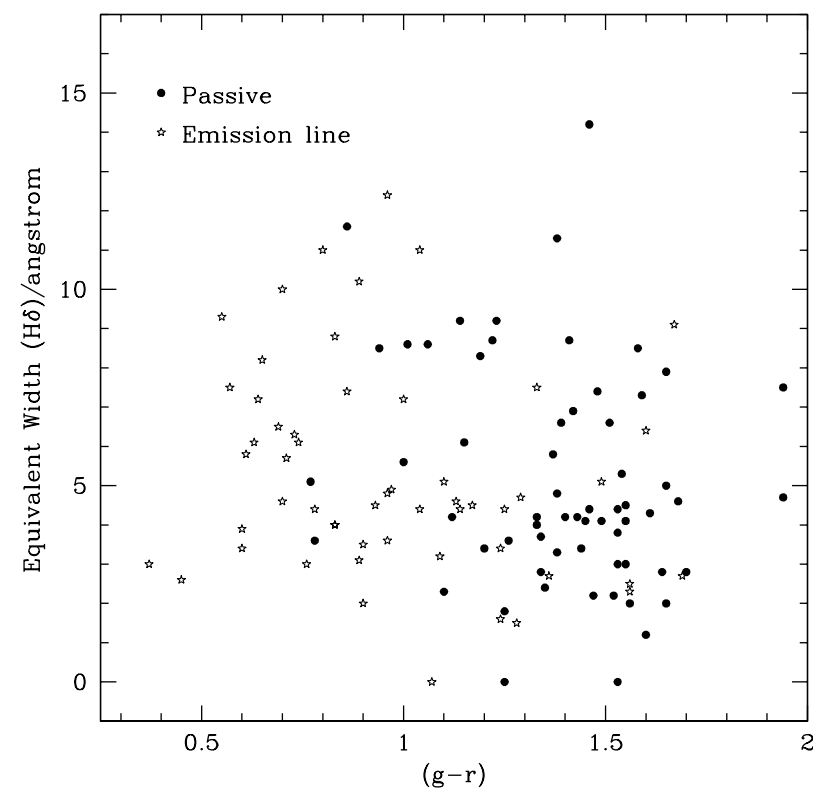

Figure 2 Distribution of cluster galaxies (mean redshift $z \sim 0.44$ ) adapted from data presented by Poggianti et al. (1999) and Dressler et al. (1999) on a $(g-r)$ colour-H $\delta$ plane. Passive galaxies are marked with filled circles and [OII] emission line galaxies with open stars.

This latter type subsequently became known as poststarbursting galaxies (PSGs) or ' $\mathrm{E}+\mathrm{A}^{\text {'1 }}$ galaxies (e.g. Franx 1993; Zabludoff et al. 1996). The presence of the $\mathrm{E}+\mathrm{A}$ galaxies in these clusters is surprising given their comparative rarity in nearby clusters (Caldwell \&

\footnotetext{
${ }^{1}$ The nomenclature ' $\mathrm{E}+\mathrm{A}$ ' derives from the dominant presence of young, luminous, blue, A-type stars (resulting from a short-lived episode of star formation) contained within a galaxy possessing an old stellar population (E). Note that Poggianti et al. (1999) have further refined this class into ' $\mathrm{k}+\mathrm{a}$ ' and ' $\mathrm{a}+\mathrm{k}$ ' species.
}

Table 1. An illustration of the morphology-density relation sourced from Oemler (1992). Because a number of sources were used in the construction of this table, the populations vary within each category and therefore these numbers should be taken as representative of the trend and not absolute. See also Figure 4 of Dressler (1980)

\begin{tabular}{lccc}
\hline Environment & \multicolumn{3}{c}{ \% Type } \\
\cline { 2 - 4 } & E & S0 & Spiral \\
\hline Field & 10 & 20 & 70 \\
Poor Group & 10 & 20 & 70 \\
Rich Group & 10 & 30 & 60 \\
Cluster & 20 & 40 & 40 \\
\hline
\end{tabular}

Rose 1997) and further reaffirms the view that clusters of galaxies have been recently evolving.

A complicating issue to the BO effect is the morphology-density $(T-\Sigma)$ relation first discussed by Dressler (1980). Table 1 provides an illustration of the $T-\Sigma$ relation sufficient for this discussion: the proportion of spiral galaxies decreases strongly as a function of local galaxy density whilst lenticular (S0) and elliptical (E) galaxies increase. Oemler (1974) demonstrates a similar result within a number of relaxed, rich clusters with the $\mathrm{E}$ and SO fraction increasing towards the centre, accompanied by a decline in the fraction of spiral types. The fraction of early types is also found to be correlated with global cluster structure: more relaxed clusters have a higher earlytype fraction (Oemler 1974). The question of whether local galaxy density or clustocentric radius is the more fundamental parameter driving the morphological transformation, however, is still an open question (Whitmore \& Gilmore 1991; Whitmore, Gilmore, \& Jones 1993).

Moreover, recent studies by Rakos, Odell, \& Shombert (1997) and Margoniner \& de Carvalho (2000) show that the bluer galaxies are indeed preferentially located in low density environs. This is particularly valid in observations of relaxed systems (e.g. Smail et al. 1998) where $f_{B}$ is computed to be smaller than that predicted by Butcher $\&$ Oemler (1984). In cases where the clusters sampled are unrelaxed or contain significant substructure, however, $f_{B}$ is found to be systematically larger (Caldwell \& Rose 1997; Metevier, Romer, \& Ulmer 2000).

\section{Discussion}

Much recent effort has gone into attempts to tie together and understand the effects outlined above. In modern times this has been facilitated with the advent of the Hubble Space Telescope (HST) and advances in computational power that permit more accurate simulations of clusters. The HST has provided an unrivalled tool for examination of galactic morphologies at high redshift.

Two groups attempted to analyse morphological information provided by the HST before its refurbishment (Couch et al. 1994; Dressler et al. 1994). Independently, they made preliminary connections between morphologically disturbed-looking galaxies and blue BO 


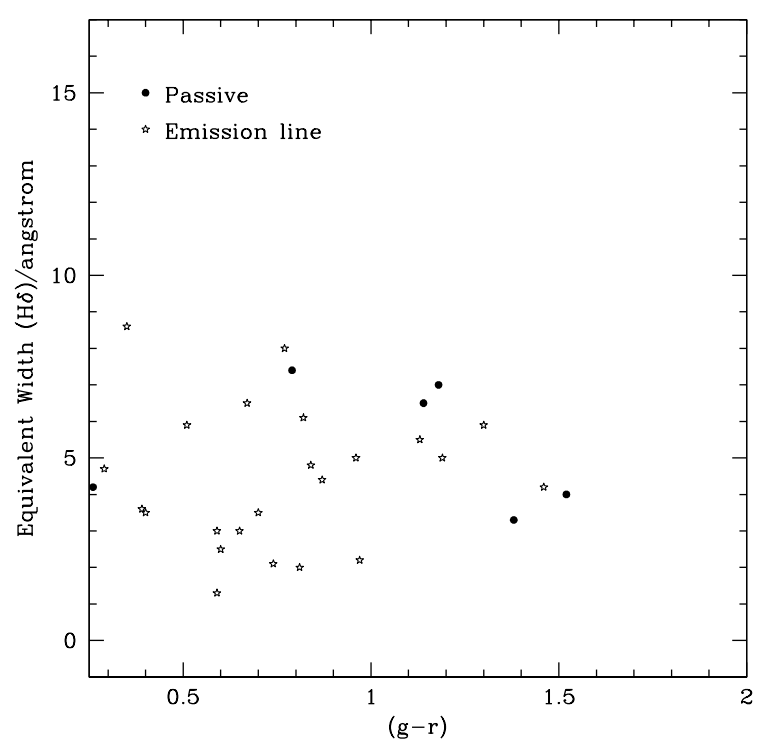

Figure 3 As for Figure 2 but for a field sample.

galaxies together with an examination of numerous elliptical galaxies possessing E+A qualities (Couch et al. 1994).

Following the refurbishment of the HST, the two groups combined their efforts in the MORPHS collaboration (Smail et al. 1997). The MORPHS sample covers 11 fields in 10 clusters in the redshift range $0.37<z<0.56$. They demonstrate that the fraction of elliptical galaxies within the rich clusters remains near constant whilst the S0 fraction decreases with increasing redshift: a redshift evolution in the $T-\Sigma$ relation (Dressler et al. 1997). Meanwhile, the $T-\Sigma$ relation is generally found to be stronger in those clusters which are more relaxed and centrally concentrated. They conclude that whilst the elliptical galaxy population would have predated the gravitational collapse of a cluster, the S0 population is only generated in large numbers after this. The blue BO galaxies, meanwhile, appear morphologically disturbed and observations of their emission lines indicate that they are indeed currently star-forming (Couch et al. 1998).

More recent work by the same collaboration suggests that recently star-forming galaxies constitute $\sim 20$ per cent of the total population (Dressler et al. 1999; Figure 2). In contrast, their incidence in the field at the same redshift is found to be lower (two per cent; Figure 3). Further, the frequency of recently star-forming galaxies belonging to clusters at these redshifts is greater than equivalent clusters at lower redshifts and has a stronger evolution with redshift than field E+As suggesting a strong evolution in this galaxy type. Those galaxies found to be star-forming (i.e. possessing emission lines) also exhibit a larger spatial extent and greater velocity dispersion than the passively evolving population. Correspondingly, the recently star-forming galaxies exhibit qualities intermediate to the star-forming and passive populations (Dressler et al. 1999; Poggianti et al. 1999).

Another major modern study of high redshift $(z=0.2-0.55)$ clusters is that of the Canadian Network for Observational Cosmology (CNOC: e.g. Yee,Ellingson,
\& Carlberg 1996; Ellingson et al. 2001). The primary CNOC sample is composed of $16 \mathrm{X}$-ray selected clusters of galaxies observed with the Canada-France-Hawai' $i$ Telescope. Over 6400 spectra have been obtained for this sample, of which over 2600 have provided a reliable redshift.

Fifteen of the CNOC clusters have been analysed by Balogh et al. (1999). In contrast to MORPHS, they find that the fraction of star-forming galaxies only weakly increases with redshift. They further find that the recently star-forming population does not increase with redshift with any significance and is not in excess to the value for the local field.

The CNOC have also examined the dependence of galaxy type with clustocentric radius: they report an absence of emission line galaxies within the cluster region correlating well with radius (Balogh et al. 1997). This correlation, however, cannot wholly be accounted for by the $T-\Sigma$ relation (Balogh et al. 1998). Several other cluster gradients are also reported by Abraham et al. (1996) and Morris et al. (1998) including a dependence of galaxy colour with clustocentric radius (i.e. environment). Work by Pimbblet et al. (2002) appears to confirm this finding with higher redshift clusters displaying a stronger radial blueing of the CMR. This can be interpreted as an age effect, whereby those galaxies at the outskirts of the cluster have younger stellar populations than those in the core region.

How is it possible to reconcile the significantly lower fraction of star-forming and post star-forming galaxies found by Balogh et al. (1997) than by Dressler et al. (1999), given that their cluster samples are coeval? A number of possibilities are explored by Ellingson et al. (2000). They attribute the discrepancies to relative differences in cluster selection technique: CNOC used X-ray selected clusters ${ }^{2}$ whereas MORPHS used optical selection. ${ }^{3}$ The CNOC spectral data also possess a lower signal-to-noise ratio than MORPHS and therefore may not be able to classify recently star-forming galaxies as accurately.

A potential complicating factor is the existence of 'dusty-starburst galaxies' reported by Poggianti et al. (1999). These galaxies exhibit both strong emission and absorption lines. It has been suggested that these potential PSG progenitors have had their star-formation rates underestimated (Poggianti et al. 1999) owing to extinction. The star-formation rate is estimated by the strength of the [OII] emission line. This line, however, is sensitive to dust extinction. Some effort is now going into using less dust-sensitive emission lines as indicators of star formation (e.g. H $\alpha$ : Balogh \& Morris 2000).

At lower redshift $(z<0.1)$ Zabludoff et al. (1996) use the Las Campanas Redshift Survey (LCRS; Shectman

\footnotetext{
${ }^{2}$ The CNOC clusters, being X-ray selected, are mostly relaxed, regular clusters, many of which contain cooling flows indicative of a lack of recent merging activity (Allen 1998).

${ }^{3}$ The MORPHS clusters were collected over a number of years during the 1990s and are composed of both optically- and AGN-selected clusters which span a range of richness and morphology.
} 
et al. 1996) to find that recently star-forming $(E+A)$ galaxies in the field constitute a low fraction $(0.2$ per cent $)$ of the total. This is in agreement with the CNOC collaboration, yet is significantly smaller than that found by the MORPHS collaboration in the field. Dressler et al. (1999) argued that the increase in recently star-forming galaxies in the field is a factor of two between their two samples.

\section{Environmental Mechanisms}

Environmental conditions can provide mechanisms to explain the observations. Assuming that initial condition considerations result in all protogalaxies being the same $(\sim$ spiral $)$, there are several processes that can take place which have the potential to explain the observations.

One classic example is that of ram pressure stripping (or ram pressure ablation: Gunn \& Gott 1972). On impact with the intra-cluster medium, galaxies that are infalling onto the cluster have their gas removed. In the case of a spiral galaxy, the stripping of its gaseous disc would result in the creation of a lenticular cluster galaxy (Quilis, Moore, \& Bower 2000). Unfortunately, this mechanism does not explain the presence of S0 galaxies in low density environments and certainly cannot be the sole cause for the observed variations in galaxy properties with environment (Lewis et al. 2002; Gómez et al. 2003).

Galaxy-galaxy interactions could also provide a mechanism for the morphological transformation of spirals into ellipticals or lenticulars (Toomre \& Toomre 1972; Barnes \& Hernquist 1991) and enhance star formation (Lavery \& Henry 1988; Lavery, Pierce, \& McClure 1992; Rakos, Maindl, \& Schombert 1996). Certainly there are enough strongly interacting galaxies observed to support this claim (Toomre 1978). Whilst this kind of interaction may explain some of the blue BO galaxies there are certainly not enough galaxy-galaxy interactions to explain all of them (Smail et al. 1997; Ghigna et al. 1998). Moore et al. (1996) propose a modified form of this interaction called 'harassment' whereby just close encounters of galaxy pairs is enough to initiate morphological transmutation.

Tidal interactions may also be taking place between galaxies and the gravitational potential of the cluster itself (Bekki, Couch, \& Shioya 2001a). This process should be a more effective mechanism than ram pressure stripping in causing activity (Byrd \& Valtonen 1990; Rose et al. 2001). Such a mechanism would, however, produce BO galaxies near the cluster core (Fujita 1998) which is observationally inconsistent.

Morphological segregation could also be driven by a slow starvation of star-forming gas (Larson, Tinsley, \& Caldwell 1980; Bekki, Couch, \& Shioya 2002). This could be caused by the removal of the gas reservoirs as galaxies enter the cluster (Poggianti et al. 1999; Balogh, Navarro, \& Morris 2000). The mechanism may lead directly to the formation of an S0 galaxy, or other processes may be involved (Balogh et al. 2001). Whatever the exact details of the process that suppresses the star formation of infalling galaxies, it necessarily must be able to operate in low density environments (Fasano et al. 2000; Bekki et al. 2001b; Carlberg et al. 2001; Couch et al. 2001; Balogh et al. 2002; also see Drinkwater et al. 2003). One problem with the starvation mechanism is the inherent gentility: it does not produce enough starbursting galaxies (Oemler 1992).

Observationally, whilst Poggianti et al. (1999) favour an abrupt truncation of the star-formation rate to explain the frequency of recently star-forming galaxies, Balogh et al. (1999) advocate a more steady decline to explain the radial dependence of emission line strength. Kodama \& Smail (2001) report that whatever the nature of the morphological transformation mechanism, the process requires $1-3$ Gyr to complete from initial infall and that clusters must necessarily have a high accretion rate.

\section{Selection Effects and Biases}

To be certain that the $\mathrm{BO}$ effect is real, one must account for the way in which cluster samples are selected. It could be that the clusters observed at higher redshift are not comparable to lower redshift ones. Oemler, Dressler \& Butcher (1997) proposed that higher redshift clusters are more exceptional than their lower redshift counterparts as they are dynamically younger and at an epoch of enhanced star formation.

Andreon \& Ettori (1999) take this thought process further. They demonstrate that the mean X-ray luminosity $\left(L_{X}\right)$ of the Butcher \& Oelmer (1984) clusters increases with redshift. Therefore, the redshift trend in $f_{B}$ might not be due to one object class. ${ }^{4}$ If true, this has implications for studies such as Ebeling, Edge, \& Henry (2001) who seek out only the most massive clusters at high redshifts. Figure 4 displays $f_{B}$ as a function of $L_{X}$ from a selection of published work. This shows no significant correlation between the two parameters.

Margoniner et al. (2001) circumvent this issue by selecting an unprecedented 295 Abell clusters (Abell 1958; Abell, Corwin, \& Olowin 1989) with no a priori bias to richness class, $L_{X}$ (and hence mass), or morphological type (and/or degree of substructure) as some other studies do (Smail et al. 1998; Balogh et al. 2002; Fairley et al. 2002; Pimbblet et al. 2002). The results of Margoniner et al. (2001) show that the evolution in $f_{B}$ is real and occurs at similar rates for clusters of all richness classes, with poorer clusters (groups) displaying higher $f_{B}$ values at any given redshift. Lastly, in contrast to Andreon \& Ettori (1999), Margoniner et al. (2001) re-affirm the view that there is no significant trend of $f_{B}$ with $L_{X}$ (see also Figure 4).

Calculation of $f_{B}$ crucially depends upon the parts of the luminosity function that are probed. Whilst Butcher \& Oemler (1984) use $M_{V}=-20$ as their fiducial magnitude cut, other investigators vary (e.g. Margoniner et al. 2001

\footnotetext{
${ }^{4}$ Cluster X-rays are produced from thermal bremsstrahlung of the (hot) inter-cluster gas (e.g. Jones \& Forman 1984; Sarazin 1988). Larger $\mathrm{X}$-ray luminosity, therefore, is indicative of more relaxed, massive clusters (Edge \& Stewart 1991; see also Zabludoff \& Zaritsky 1995 for a perspective on X-rays from a merging cluster).
} 


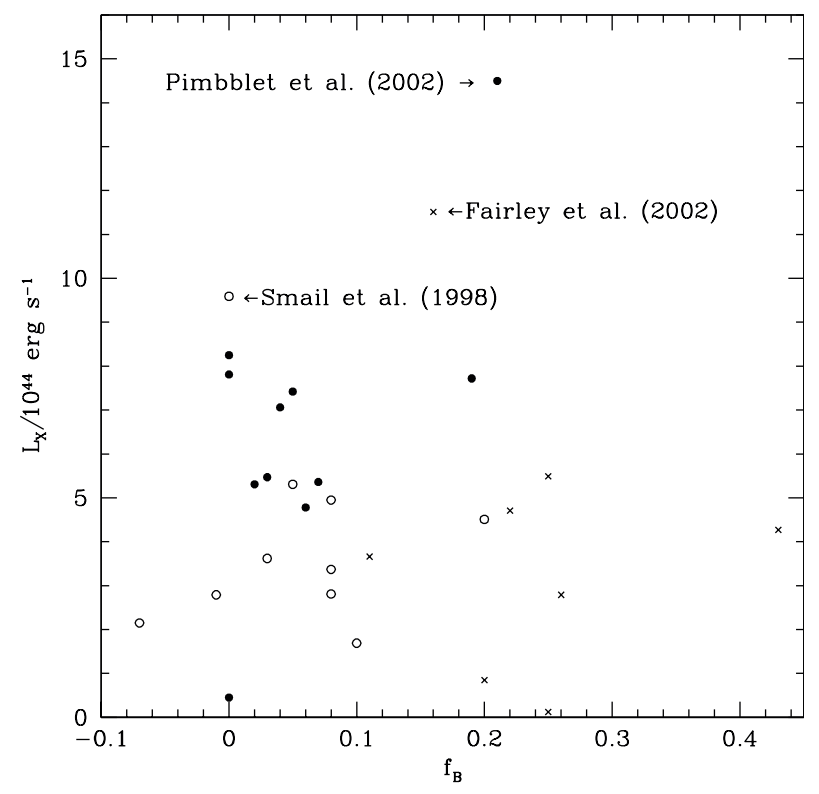

Figure 4 Blue fraction, $f_{B}$, plotted as a function of $L_{X}$ from Pimbblet et al. (2002: filled circles), Fairley et al. (2002: crosses), and Smail et al. (1998: open circles). Each set of points comes from a different redshift range $(0.08<z<0.15,0.24<z<0.58$, $0.22<z<0.28$, respectively). This is no significant correlation found between these parameters.

sample down to $M^{*}+2$ ). Sampling fainter magnitude cluster galaxies produces two major effects. Firstly, the total number of galaxies in a cluster will increase. This is beneficial as it will make Poissonian errors smaller. Secondly, different types of galaxy are sampled. This makes comparison to other works harder to facilitate as it can seriously affect the value of $f_{B}$ because fainter galaxies tend to have different colours.

Choice of the physical radius analysed (e.g. $R_{30}$ ) can also vary $f_{B}$ (see Ellingson et al. 2001). This is simple to understand in terms of the $T-\Sigma$ relation (Dressler 1980) as at larger radii, more blue, late-type galaxies will be present and cluster substructure can become important. Interestingly, by converting their selection criteria to that of the original BO work, Margoniner et al. (2001) find stronger cluster evolution than Butcher \& Oemler did. This is consistent with the results of Rakos \& Schombert (1995) and Margoniner \& de Carvalho (2000).

In studies without spectroscopic data, there will inevitably be interlopers from the field population that change $f_{B}$ (e.g. Pimbblet et al. 2002). Diaferio et al. (2001) investigated this contamination by making $N$-body simulations. Whilst interlopers may represent only a small fraction of the total cluster population, they find that they are a significant fraction (perhaps up to 50 per cent) of the bluer BO population, particularly at small clustocentric radii (i.e. within $R_{30}$ ).

\section{Concluding Remarks}

Finally, the results from two of the largest modern surveys, the 2dF Galaxy Redshift Survey (Lewis et al. 2002) and the Sloan Digital Sky Survey (Gómez et al. 2003), are proving to be interesting. Both collaborations agree that the decrease in star-formation rates of galaxies in cluster environments is a Universal phenomenon that occurs over a wide range in density. The $T-\Sigma$ relation alone is unlikely to explain the origin of this $\Sigma$ star-formation correlation (Gómez et al. 2003).

In conclusion, the landmark study of Butcher \& Oemler (1984) is merely the tip of an evolutionary iceberg incorporating photometric, spectroscopic, and morphological changes. Whilst the debate remains intense, the precise mechanism(s) required to drive these observations remains unknown. Future avenues of investigation should concentrate on a multiwavelength (wide-field) approach tied with spectroscopy (to avoid contamination effects) and must possess well defined selection criteria that can readily be compared to (or transformed directly into the selection criteria of) previous studies. One line of inquiry would be to obtain data of clusters at high redshift $(z>0.5$ and beyond) to fill in the currently sparsely-sampled region of the $f_{B}-z$ plane. Although observationally challenging, the advent of $8 \mathrm{~m}$ telescopes should make this task easier, although one must be careful in the cluster selection. Other uninvestigated areas include how $f_{B}$ varies with cluster environment within superclusters, and sampling reasonably large galaxy groups (similar to Balogh et al. 2002) to probe the extrema of the mass scale. When tied in with X-ray observations, a clearer picture of the effect that substructure plays should also become apparent.

\section{Acknowledgments}

KAP thanks the many and varied discussions with the staff at the University of Durham, plus the rest of the LARCS team. The anonymous referee also provided a number of constructive suggestions which have improved this work. This work was supported from an EPSA University of Queensland Research Fellowship.

\section{References}

Abell, G. O. 1958, ApJS, 3, 211

Abell, G. O., Corwin, H. G., \& Olowin, R. P. 1989, ApJS, 70, 1

Abraham, R. G., et al. 1996, ApJ, 471, 694

Allen, S. W. 1998, MNRAS, 296, 392

Andreon, S., \& Ettori, S. 1999, ApJ, 516, 647

Balogh, M. L., \& Morris, S. L. 2000, MNRAS, 318, 703

Balogh, M. L., Navarro, J. F., \& Morris, S. L. 2000, ApJ, 540, 113

Balogh, M. L., Christlein, D., Zabludoff, A. I., \& Zaritsky, D. 2001, ApJ, 557, 117

Balogh, M. L., Morris, S. L., Yee, H. K. C., Carlberg, R. G., \& Ellingson, E. 1997, ApJ, 488, L75

Balogh, M. L., Morris, S. L., Yee, H. K. C., Carlberg, R. G., \& Ellingson, E. 1999, ApJ, 527, 54

Balogh, M. L., Schade, D., Morris, S. L., Yee, H. K. C., Carlberg, R. G., \& Ellingson, E. 1998, ApJ, 504, L75

Balogh, M., Bower, R. G., Smail, I., Ziegler, B. L., Davies, R. L., Gaztelu, A., \& Fritz, A. 2002, MNRAS, 337, 256

Barnes, J. E., \& Hernquist, L. E. 1991, ApJ, 370, L65

Bekki, K., Couch, W. J., \& Shioya, Y. 2001a, PASJ, 53, 395

Bekki, K., Couch, W. J., \& Shioya, Y. 2002, ApJ, 577, 651

Bekki, K., Couch, W. J., Drinkwater, M. J., \& Gregg, M. D. 2001b, ApJ, 557, L39 
Bower, R. G., Lucey, J. R., \& Ellis, R. S. 1992, MNRAS, 254, 601 Butcher, H., \& Oemler, A. 1978a, ApJ, 219, 18 Butcher, H., \& Oemler, A. 1978b, ApJ, 226, 559 Butcher, H., \& Oemler, A. 1984, ApJ, 285, 426 Butcher, H. R., \& Oemler, A. 1985, ApJS, 57, 665

Butcher, H., Wells, D. C., \& Oemler, A. 1983, ApJS, 52, 183

Byrd, G., \& Valtonen, M. 1990, ApJ, 350, 89

Caldwell, N., \& Rose, J. A. 1997, AJ, 113, 492

Carlberg, R. G., Yee, H. K. C., Morris, S. L., Lin, H., Hall, P. B., Patton, D. R., Sawicki, M., \& Shepherd, C. W. 2001, ApJ, 563, 736

Couch, W. J., \& Newell, E. B. 1984, ApJS, 56, 143

Couch, W. J., \& Sharples, R. M. 1987, MNRAS, 229, 423

Couch, W. J., Ellis, R. S., Sharples, R. M., \& Smail, I. 1994, ApJ, 430,121

Couch, W. J., Barger, A. J., Smail, I., Ellis, R. S., \& Sharples, R. M. 1998, ApJ, 497, 188

Couch, W. J., Balogh, M. L., Bower, R. G., Smail, I., Glazebrook, K., \& Taylor, M. 2001, ApJ, 549, 820

Diaferio, A., Kauffmann, G., Balogh, M. L., White, S. D. M., Schade, D., \& Ellingson, E. 2001, MNRAS, 323, 999

Dressler, A. 1980, ApJ, 236, 351

Dressler, A., \& Gunn, J. E. 1983, ApJ, 270, 7

Dressler, A., Oemler, A. J., Butcher, H. R., \& Gunn, J. E. 1994, ApJ, 430, 107

Dressler, A., Smail, I., Poggianti, B. M., Butcher, H., Couch, W. J., Ellis, R. S., \& Oemler, A. J. 1999, ApJS, 122, 51

Dressler, A., et al. 1997, ApJ, 490, 577

Drinkwater, M. J., Gregg, M. D., Hilker, M., Bekki, K., Couch, W. J., Ferguson, H. C., Jones, J. B., \& Phillipps, S. 2003, Nature, 423, 519

Ebeling, H., Edge, A. C., \& Henry, J. P. 2001, ApJ, 553, 668

Edge, A. C., \& Stewart, G. C. 1991, MNRAS, 252, 414

Ellingson, E., Lin, H., Yee, H. K. C., \& Carlberg, R. G. 2001, ApJ, 547,609

Ellingson, E., Abraham, R., Yee, H. K. C., Lin, H., Laurent, G., \& Schade, D. 2000, Bulletin of the American Astronomical Society, $197,57.01$

Fairley, B. W., Jones, L. R., Wake, D. A., Collins, C. A., Burke, D. J., Nichol, R. C., \& Romer, A. K. 2002, MNRAS, 330, 755

Fasano, G., Poggianti, B. M., Couch, W. J., Bettoni, D., Kjærgaard, P., \& Moles, M. 2000, ApJ, 542, 673

Franx, M. 1993, ApJ, 407, L5

Fujita, Y. 1998, ApJ, 509, 587

Ghigna, S., Moore, B., Governato, F., Lake, G., Quinn, T., \& Stadel, J. 1998, MNRAS, 300, 146

Gómez, P. L., et al. 2003, ApJ, 584, 210

Gunn, J. E., \& Gott, J. R. I. 1972, ApJ, 176, 1

Jones, C., \& Forman, W. 1984, ApJ, 276, 38

Kodama, T., \& Smail, I. 2001, MNRAS, 326, 637

La Barbera, F., Merluzzi, P., Iovino, A., Massarotti, M., \& Busarello, G. 2003, A\&A, 399, 899
Larson, R. B., Tinsley, B. M., \& Caldwell, C. N. 1980, ApJ, 237, 692

Lavery, R. J., \& Henry, J. P. 1988, ApJ, 330, 596

Lavery, R. J., Pierce, M. J., \& McClure, R. D. 1992, AJ, 104, 2067

Lewis, I., et al. 2002, MNRAS, 334, 673

Margoniner, V. E., \& de Carvalho, R. R. 2000, AJ, 119, 1562

Margoniner, V. E., de Carvalho, R. R., Gal, R. R., \& Djorgovski, S. G. 2001, ApJ, 548, L143

Metevier, A. J., Romer, A. K., \& Ulmer, M. P. 2000, AJ, 119, 1090

Moore, B., Katz, N., Lake, G., Dressler, A., \& Oemler, A. 1996, Nature, 379, 613

Morris, S. L., Hutchings, J. B., Carlberg, R. G., Yee, H. K. C., Ellingson, E., Balogh, M. L., Abraham, R. G., \& Smecker-Hane, T. A. 1998, ApJ, 507, 84

Oemler, A. J. 1974, ApJ, 194, 1

Oemler, A. 1992, in Clusters and Superclusters of Galaxies, NATO ASIC Proceedings 366, ed. A. Fabian (Dordrecht: Kluwer Academic Publishers), 29

Oemler, A., Dressler, A., \& Butcher, H. 1997, ApJ, 474, 561

Pimbblet, K. A. 2001, PhD Thesis, University of Durham

Pimbblet, K. A., Smail, I., Kodama, T., Couch, W. J., Edge, A. C., Zabludoff, A. I., \& O'Hely, E. 2002, MNRAS, 331, 333

Poggianti, B. M., Smail, I., Dressler, A., Couch, W. J., Barger, A. J., Butcher, H., Ellis, R. S., \& Oemler, A. J. 1999, ApJ, 518, 576

Quilis, V., Moore, B., \& Bower, R. 2000, Science, 288, 1617

Rakos, K. D., \& Schombert, J. M. 1995, ApJ, 439, 47

Rakos, K. D., Maindl, T. I., \& Schombert, J. M. 1996, ApJ, 466, 122

Rakos, K. D., Odell, A. P., \& Schombert, J. M. 1997, ApJ, 490, 194

Rose, J. A., Gaba, A. E., Caldwell, N., \& Chaboyer, B. 2001, AJ, 121,793

Sarazin, C. L. 1988, X-ray Emission from Clusters of Galaxies (Cambridge: Cambridge University Press)

Shectman, S. A., Landy, S. D., Oemler, A., Tucker, D. L., Lin, H., Kirshner, R. P., \& Schechter, P. L. 1996, ApJ, 470, 172

Smail, I., Edge, A. C., Ellis, R. S., \& Blandford, R. D. 1998, MNRAS, 293, 124

Smail, I., Dressler, A., Couch, W. J., Ellis, R. S., Oemler, A. J., Butcher, H., \& Sharples, R. M. 1997, ApJS, 110, 213

Terlevich, A. I., Caldwell, N., \& Bower, R. G. 2001, MNRAS, 326, 1547

Toomre, A. 1978, in Large Scale Structures in the Universe, IAU Symposium 79, ed. M. S. Longair \& J. Einasto (Dordrecht: Reidel), 109

Toomre, A., \& Toomre, J. 1972, ApJ, 178, 623

Visvanathan, N., \& Sandage, A. 1977, ApJ, 216, 214

Whitmore, B. C., \& Gilmore, D. M. 1991, ApJ, 367, 64

Whitmore, B. C., Gilmore, D. M., \& Jones, C. 1993, ApJ, 407, 489

Yee, H. K. C., Ellingson, E., \& Carlberg, R. G. 1996, ApJS, 102, 269

Zabludoff, A. I., \& Zaritsky, D. 1995, ApJ, 447, L21

Zabludoff, A. I., Zaritsky, D., Lin, H., Tucker, D., Hashimoto, Y., Shectman, S. A., Oemler, A., \& Kirshner, R. P. 1996, ApJ, 466, 104 